\title{
Effectiveness and cost-effectiveness of a loyalty scheme for physical activity behaviour change maintenance: results from a cluster randomised controlled trial
}

Ruth F. Hunter ${ }^{1 *}$ (D) Jennifer M. Murray ${ }^{1}$, Aisling Gough ${ }^{1}$, Jianjun Tang ${ }^{1,2}$, Christopher C. Patterson?', David P. French ${ }^{3}$, Emma McIntosh ${ }^{4}$, Yiqiao Xin ${ }^{4}$, Frank Kee ${ }^{1}$ and on behalf of the Physical Activity Loyalty (PAL) Study team

\begin{abstract}
Background: We evaluated the effectiveness and cost-effectiveness of a loyalty scheme based intervention involving rewards for increasing physical activity in public sector employees.

Methods: A cluster randomised wait-list controlled trial in public sector organisations in Northern Ireland. We randomly assigned clusters (1:1) using a computer generated random sequence. Researchers were masked to allocation, but participants were not. Employees aged 18-65 years with no self-reported medical contraindications to physical activity were included. The Physical Activity Loyalty Scheme (PAL) intervention was based on high-street loyalty cards where participants earned points for minutes of activity that could be redeemed for rewards, complemented by evidence-based behaviour change techniques. The primary outcome was objectively measured mean steps/day at 6 months using a validated pedometer (Yamax Digi-Walker CW-701) over 7 days, assessed with intention to treat analysis. Secondary outcomes included health, mental wellbeing, quality of life, work absenteeism and presenteeism, and use of healthcare resources. Cost-effectiveness, cost-benefit and mediation analyses were conducted. Trial registered with Current Controlled Trials, number ISRCTN17975376.

Results: Between September 2014 and October 2015, we recruited and randomly assigned 37 clusters (from nine organisations; mean clusters per organisation $=$ four $)$ and 853 participants to the intervention $(n=19$ with 457 participants) or control group ( $n=18$ with 396 participants). Primary outcome data were available for 249 (54.4\%) intervention and 236 (59.6\%) control participants. Mean steps/day were significantly lower in the intervention vs control group (adjusted mean difference $=-336,95 \% \mathrm{Cl}:-612$ to $-60, p=0.02$ ) at 6 months. Participants redeemed only 39\% (SD 43\%) of their earned points. Using the Quality Adjusted Life Year outcome, the intervention was not cost effective from an NHS/PSS perspective. A net cost analysis from an employer perspective demonstrated the intervention group was associated with a mean of $2.97 \mathrm{~h}$ less absenteeism over a 4 week period $(p=0.62)$, which could result in net savings ranging from $£ 66$ to $£ 735$ depending on the wage rate employed. At 4-weeks postbaseline there were significant increases in identified regulation, integrated regulation, intrinsic motivation, social norms and intentions in intervention compared to control participants.

(Continued on next page)
\end{abstract}

\footnotetext{
* Correspondence: ruth.hunter@qub.ac.uk

1 UKCRC Centre of Excellence for Public Health Research (NI)/Centre for

Public Health, Queen's University Belfast, Grosvenor Road, Belfast BT12 6BJ,

Northern Ireland

Full list of author information is available at the end of the article
}

(c) The Author(s). 2018 Open Access This article is distributed under the terms of the Creative Commons Attribution 4.0 International License (http://creativecommons.org/licenses/by/4.0/), which permits unrestricted use, distribution, and reproduction in any medium, provided you give appropriate credit to the original author(s) and the source, provide a link to the Creative Commons license, and indicate if changes were made. The Creative Commons Public Domain Dedication waiver (http://creativecommons.org/publicdomain/zero/1.0/) applies to the data made available in this article, unless otherwise stated. 


\begin{abstract}
(Continued from previous page)
Conclusions: Our mixed results pose challenges that are too infrequently exposed in public heath intervention trials. Although the intervention successfully altered several hypothesised mediating constructs it did not translate into long-term behaviour change. Our incentive level may have been too low to incentivise change, despite being designed a priori by a Contingent Valuation Survey. There were also major re-structuring of several organisations which presented significant implementation challenges, and technical limitations.
\end{abstract}

Trial registration: ISRCTN17975376 (Registered 19/09/2014).

Keywords: Physical activity, Adults, Intervention, Behaviour change, Incentives, Effectiveness, Cost-effectiveness,

\section{Introduction}

There is a strong business case for investing in the health and wellbeing of the workforce [1]. It is estimated that for every $£ 1$ invested in workplace health and wellbeing, there is a potential return of over $£ 4$ as a consequence of reductions in absenteeism and improvements in productivity [2], with a possible dividend to the economy in the region of $£ 30$ billion annually [1]. With increasing numbers of inactive office-based occupations, improvements in physical activity (PA) levels may contribute positively to wellbeing, mental health, absenteeism and productivity. Evidence to support the effectiveness and cost-effectiveness of such interventions is mixed, with previous meta-analyses of workplace PA interventions showing small, positive, short-term effects on PA levels, but an absence of evidence on maintenance and cost-effectiveness from different perspectives [3-9].

The lack of evidence for the long-term effectiveness and cost-effectiveness of PA interventions is not unique to workplace interventions. A systematic review found that PA interventions targeting adults showed small positive effect sizes between 6 and 15 months follow-up (SMD 0.20-0.28), with few studies thereafter [10]. A further systematic review demonstrated that there were distinct mediators of PA behaviour change during initiation (first 6 months of a behaviour) and maintenance (beyond 6 months of a behaviour) phases [11]. This research points to the need to design interventions that purposefully support the transition from initiation to maintenance of behaviour change which involves distinct theoretical and implementation approaches.

Our earlier research demonstrated the potential effectiveness and cost-effectiveness of a workplace PA intervention in a pilot quasi-experiment, collaborating with the business sector, and purposefully developed on a sustainable 'business' model $[12,13]$. In partnership with retailers, we adapted the general underlying business principles of a loyalty scheme, an existing mechanism that supports behaviour change in the private sector, and applied it to a public health setting. In the business sector, some loyalty schemes encourage repeated behaviour (i.e., loyalty), such as shopping at a particular retailer, by rewarding participants for their repeated business by collecting points and the opportunity to convert these into subsequent rewards, such as, retail vouchers. Using similar principles, we developed the "Physical Activity Loyalty Card" whereby participants earned points for minutes of PA, which could then be redeemed for rewards. Our pilot data provided evidence to support the potential of such an intervention to impact positively on workplace health and wellbeing, and provide economic benefits from a public health, employer and retailer perspective $[12,13]$.

Consequently, using pilot data $[12,13]$ we developed a pragmatic intervention primarily aimed at increasing PA levels among office-based public sector employees in Northern Ireland [14]. The intervention incorporated evidence-based behaviour change techniques, and was underpinned by relevant theory. A cluster RCT design was employed to minimise the risk of contamination of the intervention between the trial groups. Our aims were to compare the effectiveness and cost-effectiveness of the intervention versus the waiting-list control group. A further aim was to understand the underpinning mechanisms of PA behaviour change, through process analysis of hypothesised mediating variables.

\section{Methods}

\section{Study design and participants}

The study was a cluster RCT, with a parallel mediation analysis and economic evaluation.

Nine public sector organisations in two cities (Belfast and Lisburn) in Northern Ireland were purposively sampled from those within a $2 \mathrm{~km}$ radius of the city centre or which could offer PA opportunities within a $2 \mathrm{~km}$ radius of their location and had a minimum of 100 employees in predominantly office-based occupations. Meetings were held with senior management of these organisations to explain the study purpose and practicalities.

Participants were healthy adults working in office-based occupations in public sector organisations. Recruitment methods were email invitations to employees and posters placed around each workplace advertising the study [12]. Potential participants were able to access further information (including the Participant Information Sheet) and register their interest to 
participate on the study website. They were asked to complete a screening questionnaire via the study website or by telephone, to confirm their eligibility, based on the following inclusion criteria: based at recruited worksite at least $4 \mathrm{~h} /$ day (within core hours of $8 \mathrm{am}-6 \mathrm{pm}$ ) on at least 3 days/week, current contract anticipated to last for the duration of the study, access to internet at work, able to give informed consent, and no self-reported recent medical events that would limit ability to participate in PA (assessed using the Physical Activity Readiness Questionnaire, PAR-Q).

The trial was delivered according to the updated published protocol [14]. Ethics approval was obtained from the Office for Research Ethics Committee Northern Ireland (ORECNI) (reference number 14/NI/0090; approval granted May 21, 2014). Local NHS Health and Social Care Trust approvals were obtained from Research Governance departments before the start of recruitment.

\section{Randomisation and masking}

Clusters were randomly assigned to the intervention group or waiting-list control group (1:1). Clusters were the smallest work groups or units (e.g. a large open plan office) within each participating organisation. A random allocation sequence was drawn up by the trial statistician using a computer generated random sequence, and group allocation was stratified to ensure a similar number of clusters of each size and type (i.e. small organisations <20 employees; medium organisations 20-50 employees; large organisations $>50$ employees; school) in both intervention and control groups. Allocations were only accessible by an independent trial statistician and those delivering the intervention until after the trial had finished. The outcome of the randomisation was communicated to participants by email after baseline measurements were complete.

It was not possible to blind participants to group allocation. Research staff collecting the outcome data were masked to allocation, as were the statisticians and health economists conducting the analysis (the randomisation variable was unlabelled in the main dataset).

Eligible participants were introduced to the study by the research team and gave written informed consent prior to completion of baseline data collection and before group allocation was assigned.

\section{Procedures}

Intervention components included the provision of points which could be converted into rewards (retail vouchers) contingent on meeting PA behaviour goals. The PAL Scheme integrated a PA remote tracking system with web-based monitoring and evidence-based behaviour change tools such as self-monitoring and goal-setting.
The 6 month intervention involved placing wifi beacons (sensors) at specific locations in the vicinity of participating workplaces to encourage PA within a $2 \mathrm{~km}$ radius of participants' worksites (Additional file 1). The wifi beacons were placed at locations along footpaths, in local parks, leisure centres, shopping malls, bus stops and train stations. Maps of various walking routes and details about PA opportunities tailored to the workplace were provided on the study website. Participants' PA was logged when they engaged in PA within an approximate $25 \mathrm{~m}$ radius of the wifi beacons carrying their PAL keyfob. The place, date and time of the bout of PA was logged. Participants could access their account on the study website and receive real-time feedback on the number of minutes of PA logged by the tracking system. Participants were encouraged to undertake $150 \mathrm{~min} /$ week of PA which was in line with current guidelines [15]. Minutes of PA were converted to points (1 point for $1 \mathrm{~min}$ of activity recorded), and collected points were redeemable for rewards (downloadable retail vouchers) sponsored by, and redeemable at, local businesses. To reduce the risk of 'gaming' (i.e. any items to cheat the system in order to gain more points without doing PA, for example, driving rather than walking between sensors), a daily points cap was implemented and the transit times between wifi beacons checked for anomalous values. Bonus rewards and Double Points Days were offered to participants.

Participants completed a Contingent Valuation (CV) survey [16] at baseline which provided their stated preferences to assess the minimum level of financial incentive necessary for them to increase moderate-vigorous physical activity (MVPA) (Additional file 2). This information helped determine the overall level of the rewards available for earned points. The intervention had several other components designed to enhance the effectiveness of the incentives. The techniques included the provision of regular tailored motivational emails, tailored feedback, and information on walking routes in the vicinity of the participating workplaces and links to other resources such as PA advice. It also included self-regulation techniques of goal setting, self-monitoring, and prompts to behaviour (described in detail elsewhere) [14]. These components were delivered via the study website and designed to have multiple effects: (a) to increase usage of the study website, (b) as effective behaviour change techniques in their own right, and (c) as techniques designed to aid the transition from more extrinsically motivated behaviour to more intrinsically motivated habitual behaviour.

The financial incentive component of the intervention was based on principles of Learning Theory [17] by providing an immediate reward (extrinsic motivation) for behaviours that offer health gains in the future. The use 
of contingent reinforcement hypothesised that repetition of the behaviour-reward cycle would lead to workplace PA becoming a 'learned' behaviour and lead to habit formation, which fit within a self-regulation control theory framework. Motivational messages (persuasion) and social support (vicarious experience) would increase self-efficacy satisfaction with the consequences of behaviour change acting as a reinforcing mechanism, in addition to the reinforcement of financial incentives. Thus, the financial incentive component was embedded in a complex intervention containing evidence-informed behaviour change techniques. A logic model underpinning the intervention was developed (Additional file 3).

Those assigned to the waiting-list control group $(n=$ 396) were offered the opportunity to participate in the intervention after the final follow-up period (i.e. 12 months post-baseline). Participants in this group completed outcome measures at the same time points as the intervention group but received no other intervention.

\section{Outcomes}

The primary outcome was mean steps/day (objectively measured via the validated Yamax Digi-walker CW-701 pedometer worn for seven consecutive days) for participants at the 6 month follow-up. This outcome was also collected at 12 month follow-up. Although only workplace PA was incentivised, we hypothesised that participants would be encouraged to be more active generally and so we chose a measure of total PA as our primary outcome which is most public health relevant.

Secondary outcomes at 6 month follow-up were self-reported measures of workplace PA (Global Physical Activity Questionnaire; GPAQ), health (Short Form-8), well-being (Warwick-Edinburgh Mental Well-being Scale; WEMWBS), health-related quality of life (EQ-5D-5 L), and work-related impacts (absenteeism and presenteeism using the WHO Health and Performance Questionnaire).

Hypothesised mediators of initiation and maintenance of PA were collected at baseline, at 4 weeks and 6 months post-baseline (the full list of measures is in Additional file 4).

Health-care service use and medication prescriptions were collected by self-report for the health economic evaluation.

Further details on outcome measures are provided in the Additional file 4). Self-reported outcome measures were collected at baseline and 6 months (unless otherwise stated), via online questionnaires distributed by email and automatically collated via Qualtrics (www.Qualtrics.com).

\section{Statistical analysis}

This study was powered to detect a minimum difference in means of $30 \mathrm{~min}$ of MVPA/week between groups. This is equivalent to an effect size of 0.40 assuming the outcome has a SD of 75 min. A study of 330 per group (or 660 in total) would have $90 \%$ power at the 5\% significance level to detect this difference with allowance made for an intra-cluster correlation coefficient of 0.029 , an assumed mean cluster size of 20 and a coefficient of variation in cluster size of 1.0. Assuming a 15\% drop-out, the study would therefore need to randomise 776 participants. This revised sample size was a change to protocol [14].

\section{Primary and secondary analysis}

Primary and secondary outcomes at six and 12 months post-baseline (where applicable) were compared between intervention and control groups using analysis of covariance (ANCOVAs) adjusting for baseline values, randomisation stratum (Large 50+ employees, Medium 20-50 employees, Small $<20$ employees or Schools/Colleges) and season with standard errors (SEs) and $p$-values corrected for clustering. Analysis was by intention to treat. The impact of non-response on the primary outcome findings was investigated by multiple imputation.

\section{Mediation analysis}

Hypothesised mediators of initiation and maintenance of PA behaviour change were compared between intervention and control groups using random-effects regressions. Structural equation models (SEM) were also run for all mediators of initiation and maintenance with group assignment as the independent variable, the 4 week $/ 6$ month mediator as the mediating variable and 6 month pedometer steps/day as the dependent variable. All analyses were adjusted for baseline values of the mediator and outcome, randomisation stratum and season with SEs and p-values corrected for clustering (Additional file 5). This analysis was conducted to determine whether there was a significant relationship between the mediator and outcome when controlling for group assignment.

\section{Economic evaluation}

The primary economic evaluation took the form of a within-trial cost utility analysis, adopting a public sector perspective as recommended by the UK's National Institute for Health and Care Excellence (NICE) [18] for interventions with health and non-health outcomes in the public sector (and other settings). Costs included the intervention costs (apportioned per participant) and health-care resource use. Health outcomes were expressed in terms of quality-adjusted life-years (QALYs) accrued over the 6 month follow-up period. The primary economic analysis reported an incremental cost-effectiveness ratio (ICER) estimated by dividing the adjusted difference in mean costs between groups by the adjusted difference in mean QALYs between groups. 
ICER estimates were compared with a $£ 20,000$ - $£ 30,000$ per QALY threshold applied by NICE. A supplementary Cost Benefit Analysis (CBA) was undertaken from an employer's perspective by using a 'net-cost model', incorporating the intervention costs and the avoided costs of absenteeism and productivity loss due to sick days using a human capital approach (Additional file 6).

The level of significance was $p<0.05$ for all statistical and economic analyses. Analyses were carried out using Stata release 13 (StataCorp, College Station, TX, USA).

A detailed process evaluation is published elsewhere $[19,20]$.

\section{Results}

Workplaces were recruited between September 2014 and August 2015 and participant recruitment took place between January 2015 and October 2015. A total of 1209 employees expressed an interest in participating in the study and were assessed for eligibility. Of these, 853 participants from 37 clusters were recruited and randomised into two groups ( $n=457$ intervention group, $n=$ 396 control group). The number of clusters randomised per organisation ranged from 1 to 13 , and the mean number of participants per cluster was 23 (Fig. 1). Participants redeemed 39\% (SD 43\%) of their earned points.

Table 1 shows baseline characteristics of the clusters and participants by group. The mean age of participants was 43.6 (standard deviation (SD) 9.6 ) years and $71 \%$ were female. At baseline, mean steps/day were 7826 (SD 3425).

At the 6 month follow-up, all clusters remained in the trial and we analysed 249 (54.4\%) of 457 participants in the intervention group and 236 (59.6\%) of 396 participants in the control group for the primary outcome (Fig. 1).

At 6 month post-baseline, ANCOVAs showed there was a significantly reduced mean steps/day in the intervention group relative to the control group (adjusted $b$ $=-336,95 \%$ CI: -612 to $-60, p=0.02$ adjusted for season) (Table 2). Mean steps/day decreased (from baseline) by 947 steps (SD 2702) in the intervention group, and by 398 steps (SD 2471) in the control group. The analysis was repeated following imputation of missing data by chained equations (Additional file 7). The corresponding difference in mean steps/day was -526 (95\% CI: -948 to $-104, p=0.02)$, in the same direction as the primary analysis.

At 12 months post-baseline, mean steps/day were 7790 (SD 3462) (decrease of 552 steps (SD 3183) from baseline) for the intervention group and 8203 (SD 3401) for the control group (increase of 98 steps (SD 2822) from baseline). ANCOVAs showed there was a non-significant difference between the intervention and control groups in steps/day (adjusted $b=-570,95 \% \mathrm{CI}$ : -1267 to $127, p=0 \cdot 11$ ) (Table 2).
There were significant differences between groups for self-reported workplace PA $(b-33.34,95 \%$ CI: -65.44 to 1.24, p-0.04), with workplace PA scores higher in the control group at 6 months.

At 6 months, there was a significant difference between groups for the WEMWBS ( $b=1.34,95 \%$ CI: 0.48 to $2.20, p<0.01$ ) with wellbeing scores higher in the intervention group at 6 months. After adjusting for baseline values there were no significant differences between groups for SF- 8 mental $(b=1.17,95 \%$ CI: -0.23 to 2.56 , $p=0.10)$ and physical $(b=0.64,95 \%$ CI: -0.79 to $2.08, \mathrm{p}$ $=0.38$ ) component scores, the EQ-5D-5 L health state $(b=0.70,95 \% \mathrm{CI}:-2.45$ to $3.86, \mathrm{p}=0.66)$ and weighted health index scores $(b=0.01,95 \%$ CI: $-0.01,0.04, \mathrm{p}=$ $0.37)$, and for 4 week absolute work absenteeism $(b=-$ 2.59, 95\% CI: -12.79 to $7.62, \mathrm{p}=0.62$ ), and absolute presenteeism $(b=1.48,95 \%$ CI: -0.43 to $3.38, \mathrm{p}=0.13)$ (Table 3).

Table 3 shows the absolute absenteeism hours over a 4 week period for the intervention and control group was 4.04 (SD 66.11) and 7.01 (SD 51.40) respectively ( $p=$ 0.62 ). The difference was estimated to be $2.59 \mathrm{~h}$ (adjusted) over a 4 week period $(p=0.62)$. This equates to $15.54 \mathrm{~h}$ pro-rata for a 6 month time period. After attaching the hourly salary values, the avoided cost of absenteeism and the net cost of intervention for each representative salary grade were estimated. The adjusted cost saving for the employers ranged from $£ 66-£ 735$ depending on the wage rate employed. At current intervention cost $(=£ 55.68)$, the probability that the intervention was cost-saving from the employer's perspective was $64 \%$ for the high salary group, $62 \%$ for the middle salary group, and $57 \%$ for the low salary group (Additional file 8).

Table 4 shows the results of the effects on hypothesised mediators at 4 weeks and 6 months. Additional file 9 details baseline, week four and 6 month scores on mediator variables. At 4 weeks post-baseline, there were significant differences between the intervention and control groups for intentions $(b=0.29, \mathrm{SE}=0.13, p=0.02)$, social norms $(b=0.23, \mathrm{SE}=0.08, p<0.01)$, identified regulation $(b=0.14, \mathrm{SE}=0.06, \mathrm{p}=0.01)$, integrated regulation $(b=0.23, \mathrm{SE}=0.07, \mathrm{p}<0.01)$, and intrinsic motivation $(b=0.18, \quad \mathrm{SE}=0.06, \quad \mathrm{p}<0.01)$. There were non-significant between-group differences for the other hypothesised mediators on behaviour change at 4 weeks. There were no significant associations between mediator scores and 6 month pedometer steps/day in SEM models (Additional file 9). SRMR values were close to zero for all models, and CD values ranged from 0.56-0.76.

At 6 months, there were significant differences between the intervention and control groups for identified regulation $(b=0.11, \mathrm{SE}=0.05, \mathrm{p}=0.02)$, integrated regulation $(b=0.26, \mathrm{SE}=0.08, \mathrm{p}<0.01)$, intrinsic motivation 


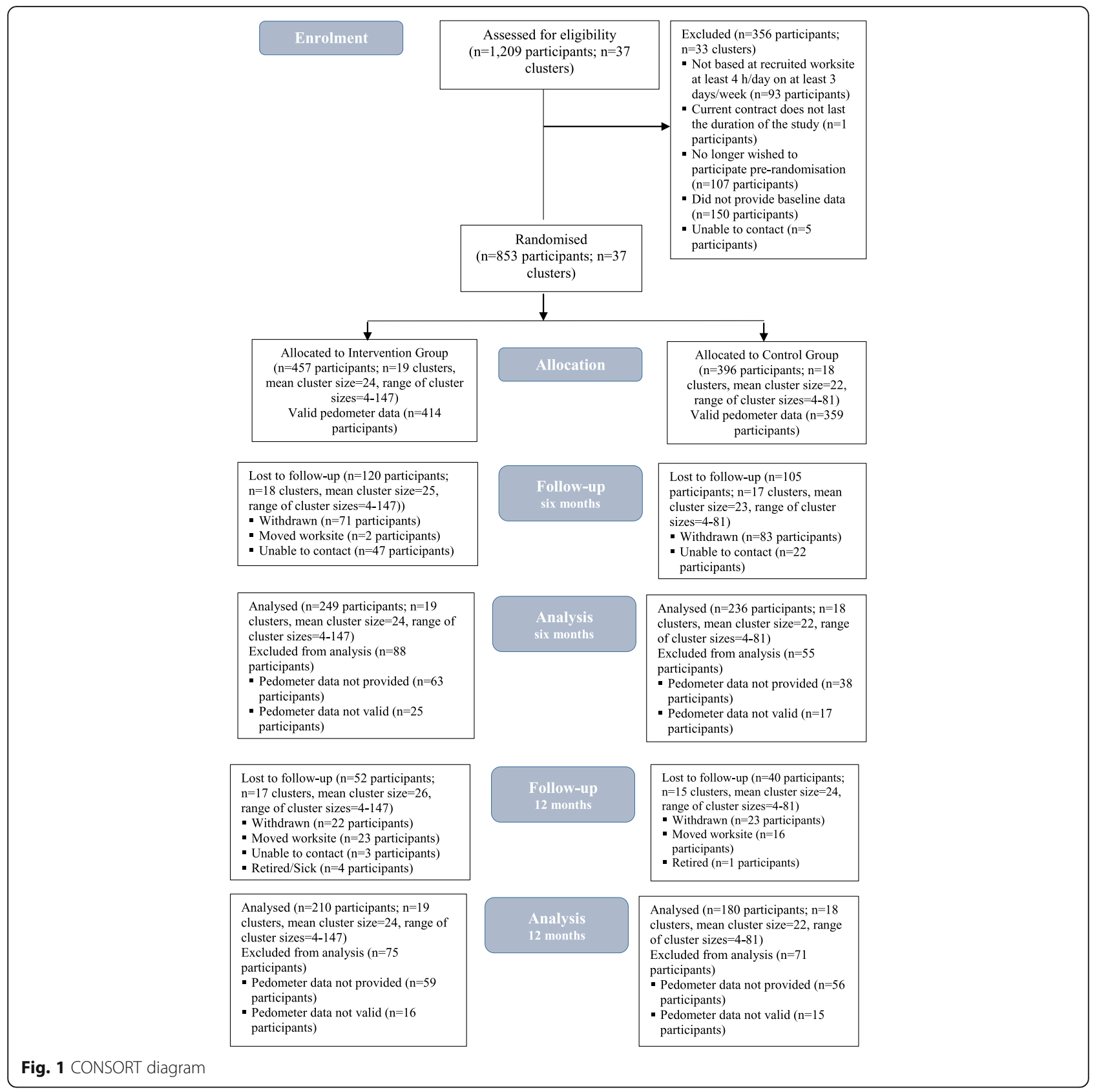

$(b=0.17, \mathrm{SE}=0.06, \mathrm{p}<0.01)$, and habit $(b=0.48, \mathrm{SE}=$ $0.12, \quad \mathrm{p}<0.01)$. There were non-significant between-group differences for the other hypothesised mediators on behaviour change at 6 months.

Results of the single mediator models with 6 month pedometer steps/day (Additional file 10) showed significant, positive intervention effects on 4 week mediator scores for intentions, social norms, identified regulation, integrated regulation and intrinsic motivation. There were no significant associations between mediator scores and 6 month pedometer steps/day. Tests of the association between mediators and PA were significant and positive for planning, social norms, identified regulation, integrated regulation, intrinsic motivation and habit. In contrast, tests of the association between mediators and PA were significant and negative for workplace norms. SRMR values were close to zero for all models, and CD values ranged from 0.62-0.76 (Additional file 10).

The intervention costs and use of health-care resources and services are detailed in the Additional files 11 and 12. Overall, there were no statistically significant differences in the quantity of each resource item between the groups; the adjusted cost of healthcare resources per participant is $£ 190.14$ for the intervention 
Table 1 Mean (SD) baseline characteristics of participants according to group

\begin{tabular}{|c|c|c|}
\hline & Intervention Group & Control Group \\
\hline Characteristics of clusters & $N=19$ & $N=18$ \\
\hline Number of participants; mean (range) & 24 (4 to 147$)$ & $22(4$ to 81$)$ \\
\hline \multicolumn{3}{|l|}{ Randomisation stratum, clusters (n, \% participants) } \\
\hline Small (<20 employees) & $11(114,25 \%)$ & $11(105,27 \%)$ \\
\hline Medium (20-50 employees) & $5(167,37 \%)$ & $4(123,31 \%)$ \\
\hline Large (> 50 employees) & $1(147,32 \%)$ & $2(144,36 \%)$ \\
\hline Schools & $2(29,6 \%)$ & $1(24,6 \%)$ \\
\hline Characteristics of participants & $n=457$ & $n=396$ \\
\hline Age (years) & $44 \cdot 0(9 \cdot 3)$ & $43.0(10.0)$ \\
\hline Female gender; $\mathrm{n}(\%)$ & $329(72 \%)$ & $278(70 \%)$ \\
\hline $\mathrm{BMI}\left(\mathrm{kg} / \mathrm{m}^{2}\right)$ & $27 \cdot 2(5 \cdot 6)$ & $26 \cdot 6(5 \cdot 3)$ \\
\hline Income >£20 k; n (\%) & $341(75 \%)$ & $291(73 \%)$ \\
\hline Education some higher level; n (\%) & $295(65 \%)$ & $270(68 \%)$ \\
\hline Marital status married/co-habiting; n (\%) & $313(68 \%)$ & $274(69 \%)$ \\
\hline Objective PA: pedometer steps (steps/day) & 7977 (3602) & $7650(3204)$ \\
\hline \multicolumn{3}{|l|}{ Objective: physical activity category, n (\%) } \\
\hline High (> 7500 steps/day) & $204(45 \%)$ & $167(42 \%)$ \\
\hline Moderate (> 2500- $\leq 7500$ steps/day) & $199(44 \%)$ & $184(46 \%)$ \\
\hline Low ( $\leq 2500$ steps/day) & $11(2 \%)$ & $8(2 \%)$ \\
\hline GPAQ: minutes of work PA (minutes/week) & $42(138)$ & $58(151)$ \\
\hline GPAQ: minutes of MVPA (minutes/week) & $296(342)$ & $344(333)$ \\
\hline \multicolumn{3}{|l|}{ GPAQ: physical activity category ${ }^{\mathrm{a}}, \mathrm{n}(\%)$} \\
\hline High & $70(15 \%)$ & $76(19 \%)$ \\
\hline Moderate & $140(31 \%)$ & $130(33 \%)$ \\
\hline Low & $141(31 \%)$ & $104(26 \%)$ \\
\hline SF-8: Mental Component Score & $48.0(8.9)$ & $47.7(9.3)$ \\
\hline SF-8: Physical Component Score & $52.5(6 \cdot 6)$ & $52 \cdot 7(7.0)$ \\
\hline EQ-5D: Health State & $82.4(13.8)$ & $83.8(14.3)$ \\
\hline EQ-5D: Weighted Health Index & $0.89(0.11)$ & $0.89(0.12)$ \\
\hline WEMWBS: Mental wellbeing scale & $50 \cdot 2(8 \cdot 2)$ & $50 \cdot 3(8.9)$ \\
\hline Physical Activity Self-Efficacy scale & $2.91(0.97)$ & $2.92(0.94)$ \\
\hline HPQ: Four week absolute absenteeism & $5.04(41 \cdot 3)$ & $3.48(50.0)$ \\
\hline HPQ: Absolute presenteeism & $80 \cdot 3(13 \cdot 6)$ & $81.0(13.4)$ \\
\hline HPQ: Combined relative absenteeism and absolute presenteeism & $8.84(12.57)$ & $8.56(7.32)$ \\
\hline
\end{tabular}

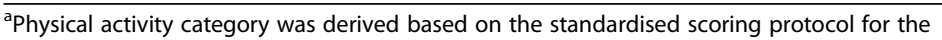
GPAQ (http://www.who.int/ncds/surveillance/steps/GPAQ\%20Instrument\%20and\%20Analysis\%20Guide\%20v2.pdf)

EQ-5D EuroQol five dimensions, GPAQ Global Physical Activity Questionnaire, $H P Q$ Health and Work Performance Questionnaire, MVPA Moderate- to vigorousintensity physical activity, NHS National Health Service, PA Physical activity, SD Standard deviation, SF Short Form, WEMWBS Warwick-Edinburgh Mental Wellbeing Scale

Please see Additional file 4 : Table S1 for further details regarding the outcome measures

group and $£ 247.70$ for the control group $(p=0.23)$. The cost utility results for the intervention are presented in Table 5. Overall, the intervention was approximately $£ 26.00$ per participant more costly but had no statistically significant effect on QALYs as compared to the control group. The average cost per participant was $£ 253.49$ (95\% CI $£ 188.41$ to 318.57 ) in the intervention group and $£ 227.64$ (95\% CI $£ 170.86$ to 284.43 ) in the control group. Mean QALYs accrued over the 6 months trial period were 0.4157 ( $95 \%$ CI 0.4077 to 0.4238 ) for the intervention group and 0.4158 (95\% CI 0.4057 to 0.4260 ) for the control group, leading to a 0.0000891 (95\% CI -0.008 to 0.008 ) lower QALY gain in the intervention group compared to the control group. A 1000 
Table 2 Mean (SD) primary outcome at 6 months and 12 months according to group and ANCOVA results before and after adjusting for season

\begin{tabular}{|c|c|c|c|c|c|c|c|c|}
\hline \multirow[t]{2}{*}{ Outcome } & \multicolumn{2}{|c|}{ Intervention Group ${ }^{a}$} & \multicolumn{2}{|c|}{ Control Group ${ }^{a}$} & \multicolumn{2}{|c|}{ Analysis of covariance ${ }^{b}$} & \multicolumn{2}{|c|}{ Analysis of covariance ${ }^{c}$} \\
\hline & $\mathrm{N}$ & Mean (SD) & $\mathrm{N}$ & Mean (SD) & $\mathrm{b}(95 \% \mathrm{Cl})$ & $\overline{p \text {-value }}$ & $\mathrm{b}(95 \% \mathrm{Cl})$ & $p$-value \\
\hline Six month: pedometer steps (steps/day) & 249 & $6990(3078)$ & 236 & $7576(3345)$ & $-519(-931,-107)$ & 0.01 & $-336(-612,-60)$ & 0.02 \\
\hline 12 month: pedometer steps (steps/day) & 210 & $7790(3462)$ & 180 & $8203(3401)$ & $-561(-1243,120)$ & 0.11 & $-570(-1267,127)$ & 0.11 \\
\hline
\end{tabular}

${ }^{\mathrm{a}}$ Month six or month 12 outcomes (unadjusted)

${ }^{\mathrm{b}}$ ANCOVA comparison of 6 month/12 month means in Intervention vs Control Group adjusted for baseline values of the outcome, and randomisation stratum and corrected for clustering

${ }^{\mathrm{C}}$ ANCOVA comparison of 6 month/12 month means in Intervention vs Control Group adjusted for baseline values of the outcome, randomisation stratum and season and corrected for clustering

ANCOVA Analysis of covariance, $\mathrm{Cl}$ confidence interval, $S D$ standard deviation

bootstrap uncertainty analysis revealed that the probability that the intervention was cost-effective at the $£ 30,000$ per QALY threshold was 34.6\%.

\section{Discussion}

In this cluster RCT, there was a significant reduction in mean steps/day (the primary outcome) in the intervention group compared to the control group at 6 months post-baseline (adjusted mean difference $=-336,95 \% \mathrm{CI}$ : -612 to $-60, p=0.02$ ). There was no significant difference between the intervention group and the control group in mean steps/day at 12 months post-baseline (adjusted mean difference $=-570,95 \% \mathrm{CI}:-1267$ to $127, \mathrm{p}=0 \cdot 11$ ).

By contrast, the study found significant intervention effects for improvement in mental wellbeing. However, this was not supported with findings from the quality of life and mental health measures, and so we are cautious in over-interpreting this positive finding. It is conceivable that mental wellbeing improved in the intervention group as a result of being involved in an employee health and wellbeing trial at a time when there were significant re-structuring in the organisations.

Further, there was a $60 \%$ probability of the intervention being cost-saving from an employer's perspective arising from reduced absenteeism $(\mathrm{p}=0.62)$. Whilst the decline in absenteeism hours was not statistically significant it is arguably economically significant and worth exploring in future studies powered to detect such economic impacts [21]. Hence, from the employers perspective and valuing absenteeism using a human capital approach, the intervention could be deemed to be worthwhile.

Table 3 Mean (SD) secondary outcomes at 6 months according to group and ANCOVA results before and after adjusting for season

\begin{tabular}{|c|c|c|c|c|c|c|c|c|}
\hline \multirow[t]{2}{*}{ Outcome } & \multicolumn{2}{|c|}{$\begin{array}{l}\text { Intervention } \\
\text { Group }^{\mathrm{a}}\end{array}$} & \multicolumn{2}{|c|}{ Control Group ${ }^{a}$} & \multicolumn{2}{|c|}{ Analysis of covariance ${ }^{b}$} & \multicolumn{2}{|c|}{ Analysis of covariance ${ }^{c}$} \\
\hline & $\mathrm{n}$ & Mean (SD) & $\mathrm{n}$ & Mean (SD) & $\mathrm{b}(95 \% \mathrm{Cl})$ & $\begin{array}{l}p^{-} \\
\text {value }\end{array}$ & $\mathrm{b}(95 \% \mathrm{Cl})$ & $\begin{array}{l}\mathrm{p}^{-} \\
\text {value }\end{array}$ \\
\hline GPAQ: minutes of work PA (minutes/week) & 253 & $16(58)$ & 235 & $44(129)$ & $-34(-65,-2$ & 0.04 & $-33(-65,-1)$ & 0.04 \\
\hline GPAQ: minutes of MVPA (minutes/week) & 231 & $\begin{array}{l}291 \cdot 8 \\
(255 \cdot 8)\end{array}$ & 221 & $\begin{array}{l}350 \cdot 1 \\
(329 \cdot 4)\end{array}$ & $\begin{array}{l}-2 \cdot 8(-63 \cdot 4 \\
57 \cdot 9)\end{array}$ & 0.93 & $4 \cdot 1(-47 \cdot 1,55 \cdot 3)$ & $0 \cdot 88$ \\
\hline SF-8: Mental Component Score & 269 & $48.4(8.9)$ & 244 & $47 \cdot 1(9.4)$ & $1 \cdot 1(-0.3,2 \cdot 4)$ & 0.12 & $1 \cdot 2(-0 \cdot 2,2 \cdot 6)$ & $0 \cdot 10$ \\
\hline SF-8: Physical Component Score & 269 & $50 \cdot 9(8.4)$ & 244 & $51 \cdot 0(7 \cdot 6)$ & $0.43(-1 \cdot 3,2 \cdot 1)$ & 0.62 & $0 \cdot 6(-0 \cdot 8,2 \cdot 1)$ & 0.38 \\
\hline EQ-5D: Health State & 262 & $78.0(16 \cdot 2)$ & 239 & $78.3(16 \cdot 1)$ & $0.7(-2 \cdot 4,3 \cdot 7)$ & 0.66 & $0.70(-2.5,3.9)$ & 0.66 \\
\hline EQ-5D: Weighted Health Index & 262 & $0.8(0 \cdot 1)$ & 239 & $0.8(0.2)$ & $\begin{array}{l}0.01(-0.02, \\
0.03)\end{array}$ & 0.71 & $\begin{array}{l}0.01(-0.01 \\
0.04)\end{array}$ & 0.37 \\
\hline WEMWBS: Mental wellbeing scale & 266 & $50 \cdot 3(8.4)$ & 243 & $49.4(8.2)$ & $1 \cdot 2(0 \cdot 2,2 \cdot 1)$ & 0.02 & $1 \cdot 3(0.5,2 \cdot 2)$ & $<0.01$ \\
\hline HPQ: Four week absolute absenteeism & 247 & $4 \cdot 0(66 \cdot 1)$ & 227 & $7.0(51.4)$ & $\begin{array}{l}-2 \cdot 25(-12 \cdot 5 \\
8 \cdot 0)\end{array}$ & 0.67 & $\begin{array}{l}-2.59(-12 \cdot 8 \\
7 \cdot 6)\end{array}$ & 0.62 \\
\hline HPQ: Absolute presenteeism & 261 & $78.6(14 \cdot 6)$ & 236 & $78.5(14 \cdot 2)$ & $0.8(-1 \cdot 8,3 \cdot 4)$ & 0.54 & $1.5(-0.4,3.4)$ & 0.13 \\
\hline $\begin{array}{l}\text { HPQ: Combined relative absenteeism and absolute } \\
\text { presenteeism }\end{array}$ & 246 & $9.5(24 \cdot 6)$ & 226 & $7.9(4.9)$ & $\begin{array}{l}-0.03(-0.9 \\
0.9)\end{array}$ & 0.95 & $\begin{array}{l}-0.02(-0.9, \\
0.9)\end{array}$ & 0.96 \\
\hline
\end{tabular}

a Month six outcomes (unadjusted)

${ }^{\mathrm{b}}$ ANCOVA comparison of 6 month means in Intervention vs Control Group adjusted for baseline values of the outcome, and randomisation stratum and corrected for clustering

CANCOVA comparison of 6 month means in Intervention vs Control Group adjusted for baseline values of the outcome, randomisation stratum and season and corrected for clustering

ANCOVA Analysis of Covariance, $C l$ confidence interval, EQ-5D EuroQol five dimensions, GPAQ Global Physical Activity Questionnaire, MVPA moderate- to vigorousintensity physical activity, NHS National Health Service, PA physical activity, SD standard deviation, SE standard error, SF Short Form, WEMWBS Warwick-Edinburgh Mental Wellbeing Scale 
Table 4 Effects of intervention on hypothesised mediators, at 4 weeks or 4 months, with adjustment for baseline values (coefficients and standard errors from random-effects regressions)

\begin{tabular}{|c|c|c|c|c|c|c|}
\hline \multirow[t]{2}{*}{ MEDIATOR (range) } & \multicolumn{3}{|c|}{ Four week mediators } & \multicolumn{3}{|c|}{ Six month mediators } \\
\hline & $n$ & $b(S E)$ & p-value & $n$ & $b(S E)$ & $\mathrm{p}$-value \\
\hline PA self-efficacy(1-5) & 597 & $0.09(0.08)$ & 0.31 & & & \\
\hline Intentions (1-7) & 595 & $0.29(0.13)$ & 0.02 & & & \\
\hline Outcome expectations (1-5) & 528 & $-0.03(0.06)$ & 0.58 & & & \\
\hline Financial motivation (1-7) & 600 & $0.11(0.15)$ & 0.46 & & & \\
\hline Planning (1-4) & 575 & $0.02(0.05)$ & 0.75 & 436 & $0.08(0.06)$ & $0 \cdot 18$ \\
\hline Social norms (1-7) & 576 & $0.23(0.08)$ & $<0.01$ & 434 & $0.10(0.12)$ & 0.42 \\
\hline Identified regulation (1-5) & 598 & $0.14(0.06)$ & 0.01 & 459 & $0.11(0.05)$ & 0.02 \\
\hline Integrated regulation (1-5) & 595 & $0.23(0.07)$ & $<0.01$ & 454 & $0.26(0.08)$ & $<0.01$ \\
\hline Intrinsic motivation (1-5) & 599 & $0.18(0.06)$ & $<0.01$ & 456 & $0.17(0.06)$ & $<0.01$ \\
\hline Habit (1-5) & & & & 448 & $0.48(0.12)$ & $<0.01$ \\
\hline Workplace norms (1-5) & & & & 456 & $0.13(0.07)$ & 0.06 \\
\hline Recovery self-efficacy (1-4) & & & & 457 & $0.02(0.06)$ & $0 \cdot 80$ \\
\hline Maintenance self-efficacy (1-4) & & & & 459 & $-0.02(0.09)$ & 0.80 \\
\hline Outcome satisfaction (1-5) & & & & 427 & $0.03(0.05)$ & 0.56 \\
\hline
\end{tabular}

PA Physical activity, SE Standard error

NB. All questionnaire items were scaled so that lower values indicated lower levels of the mediator/outcome. Results are adjusted for strata, season, baseline pedometer steps/day and baseline mediator values with cluster-adjusted standard errors and $p$-values $(b=$ coefficient for group assignment variable, i.e. Intervention versus Control)

Our analyses included an adjustment for season which would suggest that the 6 month difference in steps/day between the intervention and control groups was not due to a seasonal effect. Given the rationale for intervention, its theoretical underpinning and our pilot data, these results defy easy explanation. Our qualitative findings point to some issues with the level and type of incentives [19]. Participants redeemed 39\% (SD 43\%) of their earned points. These aspects were informed a priori via a CV survey with all participants, and with the types of vouchers were discussed in pre-intervention focus groups with the target population. They were also shown to be popular in our pilot work [12]. Our intervention was 6 months in duration which is in line with other PA interventions attempting to elicit and support PA maintenance (a period consonant with the mean in a recent review [10]). Usage data from the PA monitoring system showed that the minutes of activity recorded on the system declined over the 6 month intervention period (participants logging at least 10 min of activity via the PA monitoring system on $25 \%$ of all possible intervention days). However, from our qualitative findings it is clear that some participants reported feeling frustrated with early technological glitches that impacted on accurate monitoring of PA behaviour [19, 20]. New rewards, walking routes, and double point's days were regularly introduced in an attempt to keep the format fresh and appealing but this did not improve workplace PA. The

Table 5 Cost-effectiveness results (incremental cost per QALY) with 6 month follow-up

\begin{tabular}{|c|c|c|c|c|}
\hline \multirow[t]{2}{*}{ Treatment group } & \multicolumn{2}{|c|}{ Cost $(£)^{a}$} & \multicolumn{2}{|l|}{ QALY } \\
\hline & Mean & $95 \% \mathrm{Cl}$ & Mean & $95 \% \mathrm{Cl}$ \\
\hline Intervention $(n=457)$ & 253.49 & $188 \cdot 41,318.57$ & 0.4157 & $0.4077,0.4238$ \\
\hline Control $(n=396)$ & $227 \cdot 64$ & $170 \cdot 86,284 \cdot 43$ & $0 \cdot 4158$ & $0.4057,0.4260$ \\
\hline Difference $(95 \% \mathrm{Cl})$ & $25 \cdot 85$ & $-29 \cdot 89,81 \cdot 60$ & -0.0000891 & $-0.008,0.008$ \\
\hline ICER & \multicolumn{4}{|c|}{$-£ 290,178$ per QALY } \\
\hline 95\% CI for ICER (from bootstrap) & \multicolumn{4}{|c|}{ - $£ 480,012$ to $-£ 100,336$ per QALY } \\
\hline
\end{tabular}

${ }^{a}$ Adjusted cost, per-participant intervention cost included in the cost of intervention group

${ }^{\mathrm{b}}$ Adjusted QALY

Costs were estimated using generalised linear models, gamma family, log link. Decrements of QALY were estimated using generalized linear models, gamma family, log link. Arm level costs and QALYs were obtained from recycled predictions. Covariates for the models were: baseline cost and baseline utility, mean steps, age, sex, SF-8 physical and mental scores, cluster, Strata, and season. 95\%Cls were obtained from 1000-iteration bootstrap CI Confidence interval, ICER Incremental cost-effectiveness ratio, QALY Quality Adjusted Life Year, SE Standard error 
importance of study context should not be underestimated in the interpretation of our study findings. During our recruitment phase, a number of participating organisations undertook significant re-structuring due to the then current economic austerity, resulting in uncertainty regarding job security and job location; a time when employee health and wellbeing was at its most vulnerable. The impact of this was evident in our qualitative data which highlighted how motivation can be more usefully seen as a property of systems (incorporating technologies, organisation and action) rather than just of individuals [19]. Further details regarding the findings from the process evaluation are provided in Gough et al. (2018) [19] and Murray et al. (2019) [20].

Our findings suggest interesting conjectures regarding causal mechanisms. The use of financial incentives to change health behaviours is often criticised for 'crowding out' intrinsic motivation in line with Self Determination Theory - albeit based on laboratory based experiments [22]. Findings from our 'real-world' study provide evidence to the contrary in two ways: (1) our results showed increased internal forms of motivation (i.e. identified regulation, integrated regulation and intrinsic motivation) for the intervention group compared to the control group at 4 weeks and 6 months; (2) results demonstrated that there was no intervention effect for financial (extrinsic) motivation at 4 weeks. These findings have important implications in respect of some of the contentious issues highlighted in the literature on the use of financial incentives for achieving behaviour change. They suggest that using financial incentives within a complex behaviour change intervention with multiple components collectively does not necessarily diminish, and may facilitate intrinsic motivation. Our results also suggest that the provision of financial incentives does not necessarily increase financial (extrinsic) motivation. These findings are in line with a systematic review of psychological and economic studies which concluded that there was no evidence that extrinsic incentives would crowd out incentivised health behaviours [23]. However, given the complex nature of this intervention, it is important to note that the positive findings for internal motivation could also be related to our use of self-regulation Behaviour Change Techniques including self-monitoring and feedback on PA behaviour.

More generally, whilst the intervention group showed increases in some hypothesised mediators of initiation, these increases were not related to PA behaviour at 6 months. However, hypothesised mediators of maintenance were related to PA behaviour at 6 months. Especially notable are the findings that internal motivation mitigated reduction in PA behaviour at 6 months. Self-regulation appears to have mitigated and attenuated the reduction in steps in the intervention group. As reported above, our results run counter to predictions of Self Determination Theory suggesting that there is a role for financial incentives and self-regulation interventions. However, the amount of variance explained by our measured mediators of behaviour change was low. Therefore, future studies would need to examine other potential mediators of behaviour change to shed further light on these associations. Our previous systematic review [11] suggested that studies of mechanisms of intervention effect have generally been of poor methodological quality and would benefit from a framework based on consensus about how mediation should be measured and tested in trials of complex interventions. Such a framework should include the use of formal mediation tests, the embedding of evidence-based techniques for changing hypothesised mediators and the need to investigate constructs with particular relevance for initiation and maintenance of behaviour change. Future research should also examine mediators of adverse effects such as found in the present study so we can better understand unintended consequences and negative findings [24].

Despite the improvements in some secondary outcomes and positive net-benefit for employers, the primary outcome (steps/day) declined significantly in the intervention group. These results pose several scientific and real world implementation challenges that are too infrequently exposed in public heath intervention trials [25], including how to balance positive and negative results when primary and secondary outcomes are discordant [26].

There is a long standing belief that positive results are favoured by scientific journals and that this may contribute to "publication bias". On the other hand, some journals claim now to select articles for publication based on their contribution to the literature and welcome null results that challenge conventional wisdom or prior expectations [27]. The results from our trial certainly challenged prior expectations. However, it is notoriously hard to disprove any hypothesis, and so negative studies must have the precision and strength of design to be reasonably persuasive. The revised sample size and power calculations for our trial were approved by the study funder, and the effect size on which they were premised was within the category of "moderate" effect sizes observed in past PA interventions [28]. Even though attrition and loss to follow-up was higher than predicted (43\% observed vs $15 \%$ predicted), (albeit comparable to literature published during the conduct of our trial.) [29] we might have concluded that accepting the null hypothesis at 12 months was justified. However, the primary outcome was specified at 6 months and the non-significant difference in mean steps/day at 12 months ( 500 steps) was, arguably, still of a magnitude 
which could be of public health importance. We would probably have claimed as much had the direction of the intervention effect been positive. Some may also argue that with a primary outcome of total PA, the lack of a significant intervention effect is not surprising given that the intervention specifically incentivised workplace PA. However, we hypothesised that participants would also be encouraged to further participate in additional PA outside the workplace. Workplace PA was specifically measured using the remote sensing monitoring system (sensors and keyfobs) and the GPAQ which incorporates a workplace PA domain. However, we also used a well-validated pedometer, followed a standardised measurement protocol (including sealing the pedometer to prevent reactivity), and we supplemented the data with the GPAQ and daily PA monitoring using the remote sensing system. There was consistency in the direction and magnitude of our findings for total PA and workplace PA.

If the significant negative effect at 6 months is indicative of a true negative effect of this intervention in this setting, one might still ask whether the result is generalisable. Is this recruited sample of public sector office workers in Northern Ireland representative of office workers elsewhere in the UK? We have no reason to conclude that it is not $[3,5,7,13]$, but are mindful of the modelling undertaken by Basu and Kiernan [30] which demonstrated that two key factors impacting the success of workplace-based financial incentives for behaviour change are (i) who participates (this will be explored in a detailed process evaluation as the overall null effect might be masking differential effects in different population sub-groups) and (ii) the levels of incentives. Thus it is important to further investigate the potential causal mechanisms using mediation and moderation analyses to gain a better understanding of our findings. Our study was not powered to detect changes in mediating variables, and because of low power and multiple testing, the results need to be interpreted with caution.

\section{Conclusions}

In summary, the PAL Scheme intervention was not more effective than waiting-list control. Reduced health care costs, reduced absenteeism and improved mental wellbeing in the intervention group are somewhat noteworthy, and results suggest that the intervention could be cost beneficial for employers. However this needs to be explored within a trial powered on the economic outcome of productivity and/or absenteeism. Finally, we believe our results pose several scientific and real world implementation challenges that are too infrequently exposed in public heath intervention trials.

\section{Additional file} Additional file 1: Components of the physical activity monitoring
system (wifi beacons, keyfobs and website). (DOCX $1236 \mathrm{~kb}$ )

Additional file 2: Methodology: Contingent Valuation Survey to elicit plausible financial incentives required for increasing physical activity. (DOCX $20 \mathrm{~kb}$ )

Additional file 3: Figure S1. Logic model of the Physical Activity Loyalty scheme. (DOCX 62 kb)

Additional file 4: Table S1. Description of the primary and secondary outcomes and measures. (DOCX 27 kb)

Additional file 5: Methodology-Mediation analyses. (DOCX $21 \mathrm{~kb}$ ) Additional file 6: Methodology-Economic evaluation. (DOCX $23 \mathrm{~kb}$ )

Additional file 7: Table S2. Mean (SD) outcomes at months six and 12 according to group and ANCOVA results before and after adjusting for season, with imputation of missing values on the six or 12 month outcomes. (DOCX $20 \mathrm{~kb}$ )

Additional file 8: Table S3. Estimation of avoided cost of absenteeism and net cost of intervention using the 'net cost model'. (DOCX $20 \mathrm{~kb}$ )

Additional file 9: Table S4. Baseline, four week and six month scores on mediator variables. (DOCX $23 \mathrm{~kb}$ )

Additional file 10: Table S5. Results of single mediator models with six month pedometer steps/day as the dependent variable. (DOCX 24 kb)

Additional file 11: Table S6. Intervention costs. (DOCX 21 kb)

Additional file 12: Table S7. NHS and social care resource use per participant over six months (complete case). (DOCX 24 kb)

\section{Abbreviations}

ANCOVA: Analysis of Covariance; BMI: Body Mass Index; CBA: Cost Benefit Analysis; CD: Coefficient of Determination; Cls: Confidence Intervals;

CV: Contingent Valuation; EQ-5D: Euroqol 5 dimension; EQ-5D-5 L: Euroqol 5 dimension, 5 level; GPAQ: Global Physical Activity Questionnaire;

ICER: Incremental Cost-Effectiveness Ratio; ISRCTN: International Standard Randomised Controlled Trial Number; MVPA: Moderate-to-Vigorous Physical Activity; NHS: National Health Service; NICE: The National Institute for Health and Care Excellence; NIHR: National Institute for Health Research;

ORECNI: Office of Research Ethics Committees Northern Ireland; PA: Physical Activity; PAL: Physical Activity Loyalty; PAR-Q: Physical Activity Readiness Questionnaire; PHR: Public Health Research; PSSRU: Personal Social Service Research Unit; QALY: Quality Adjusted Life Year; RCT: Randomised Controlled Trial; SD: Standard Deviation; SE: Standard Errors; SEM: Structural Equation Modelling; SF-8: Short Form 8; SMD: Standardised Mean Difference; SRMR: Standardised Root Mean Square Residual; WEMWBS: WarwickEdinburgh Mental Wellbeing Scale; WHO: World Health Organization

\section{Acknowledgements}

This work was supported by the National Institute for Health Research Public Health Research Programme under Grant number 12/211/82. RH is supported by a NIHR Career Development Fellowship (CDF-2014-07-020) and acknowledges funding support from the HSC Research and Development Division. JM was supported by a PhD studentship from the Department for the Economy (Northern Ireland).

The work was undertaken under the auspices of the UKCRC Centre of Excellence for Public Health Research Northern Ireland and Centre for Diet and Activity Research (CEDAR), UKCRC Public Health Research Centres of Excellence which are funded by the British Heart Foundation, Cancer Research UK, Economic and Social Research Council, Medical Research Council, the National Institute for Health Research, and the Wellcome Trust. The study team would also like to acknowledge the Institute of Electronics, Communications and Information Technology (ECIT), Queen's University Belfast, Club Marketing Services Ltd., Lisburn City Centre Management, and support from project partners, the South Eastern Health and Social Care Trust, and Lisburn and Castlereagh City Council. The authors also wish to acknowledge Jennifer Badham, Queen's University Belfast for her assistance with data cleaning. 
The views and opinions expressed therein are those of the authors and do not necessarily reflect those of the Public Health Research programme, NIHR, NHS or the Department of Health.

\section{Funding}

The study was funded by the National Institute for Health research (NIHR) Public Health Research (Ref: 12/211/82). The funder of the study had no role in study design, data collection, data analysis, data interpretation, or writing of the report.

\section{Availability of data and materials}

The datasets used and/or analysed during the current study are available from the corresponding author on reasonable request.

\section{Authors' contributions}

$\mathrm{RH}$ was joint Principal Investigator, led the design and execution of the study, was involved in the interpretation of the data, and led the writing of the manuscript. JM assisted with recruitment and data collection, led the analyses for the primary and secondary outcomes, and the mediation analyses, was involved in the interpretation of the data, and was involved in drafting the manuscript. AG was the project manager and led the six and 12 month data collection process and running of the trial, was involved in interpretation of the data and drafting of the manuscript. JT led the conduct, analysis and interpretation of the CV survey that informed the level of financial incentive used in the intervention, and assisted with recruitment and data collection. CP supervised the design, conduct and interpretation of the quantitative data for the primary and secondary analysis. DF oversaw the theoretical aspect of the intervention, advised on the measurement of mediators of behaviour change, and was significantly involved in the interpretation of the mediation analyses. EMcl supervised the design, conduct and interpretation of the health economic data, including the costbenefit analyses. $\mathrm{XY}$ led the health economic analyses, and was significantly involved in the interpretation of the data, and wrote the health economic material and supplementary material. FK was the joint Principal Investigator, led the design and execution of the study, oversaw all subsequent analyses and interpretation of the data, and was involved in drafting the manuscript. The authors wish to acknowledge the significant contribution of the PAL Study team including: Sarah F. Brennan (project manager year 1-2 of the study), Alberto Longo and George Hutchinson (developed the CV survey and oversaw the conduct and interpretation of the results), Lindsay Prior (advised on the qualitative research), Mark A. Tully (advised on physical activity measurement) from Queen's University Belfast; Jean Adams (advised on the financial incentive framework), University of Cambridge; Oliver J. ChrzanowskiSmith (Research Assistant year 1-3 of the study), University of Bath; Angela Carlin (Research Assistant year 4 of the study), Ulster University; Nikki Boyer (Health economics Research Assistant year 1-2 of the study), University of Glasgow. All authors contributed to study design and intervention development. All authors reviewed and interpreted the result and edited the manuscript. All authors read and approved the final manuscript.

\section{Ethics approval and consent to participate}

Ethics approval was obtained from the Office for Research Ethics Committee Northern Ireland (ORECNI) (reference number 14/NI/0090; approval granted May 21, 2014). Local NHS Health and Social Care Trust approvals were obtained from Research Governance departments before the start of recruitment. All participants provided written informed consent prior to the start of the study.

\section{Consent for publication}

Not applicable.

\section{Competing interests}

Prof Frank Kee, Prof Emma McIntosh and Prof David French are members of the PHR Funding Board. Prof Frank Kee is also a member of the PHR Prioritisation Group.

\section{Publisher's Note}

Springer Nature remains neutral with regard to jurisdictional claims in published maps and institutional affiliations.

\section{Author details}

'UKCRC Centre of Excellence for Public Health Research (NI)/Centre for Public Health, Queen's University Belfast, Grosvenor Road, Belfast BT12 6BJ, Northern Ireland. ${ }^{2}$ School of Agricultural Economics and Rural Development, Renmin University of China, Beijing, China. ${ }^{3}$ School of Health Sciences, University of Manchester, Manchester, England. ${ }^{4}$ Health Economics and Health Technology Assessment, University of Glasgow, Glasgow, Scotland.

Received: 20 August 2018 Accepted: 25 November 2018

Published online: 12 December 2018

\section{References}

1. Working for a healthier tomorrow. Presented to the Secretary of State for Health and the Secretary of State for Work and Pensions Dame Carol Black's Review of the health of Britain's working age population 17th March 2008. Available at: https://www.gov.uk/government/publications/working-for-ahealthier-tomorrow-work-and-health-in-britain. Accessed 28 Mar 2018.

2. Price Waterhouse Coopers. Building the case for wellness. 2008. Available at: https://www.gov.uk/government/publications/work-health-and-wellbeingbuilding-the-case-for-wellness. Accessed 28 Mar 2018.

3. Abraham C, Graham-Rowe E. Are worksite interventions effective in increasing physical activity? A systematic review and meta-analysis. Health Psychol Rev. 2009;3:108-44.

4. Conn VS, Hafdahl AR, Cooper PS, Brown LM, Lusk SL. Meta-analysis of workplace physical activity interventions. Am J Prev Med. 2009;37:330-9.

5. Hutchinson AD, Wilson C. Improving nutrition and physical activity in the workplace: a meta-analysis of intervention studies. Health Promot Int. 2012;27: 238-49.

6. Freak-Poli RL, Cumpston M, Peeters A, Clemes SA. Workplace pedometer interventions for increasing physical activity. Cochrane Database Syst Rev. 2013;4:CD009209. https://doi.org/10.1002/14651858.CD009209.pub2.

7. Rongen A, Robroek SJ, van Lenthe FJ, Burdorf A. Workplace health promotion: A meta-analysis of effectiveness. Am J Prev Med. 2013:44(4):406-15.

8. Malik S, Blake H, Suggs S. A systematic review of workplace health promotion interventions for increasing physical activity. Br J Health Psychol. 2014;19:149-80.

9. Abdin S, Welch RK, Byron-Daniel J, Meyrick J. The effectiveness of physical activity interventions in improving well-being across office-based workplace settings: a systematic review. Public Health. 2018;160:70-6.

10. Murray JM, Brennan SF, French DP, Patterson CC, Kee F, Hunter RF. Effectiveness of physical activity interventions in achieving behaviour change maintenance in young and middle aged adults: a systematic review and meta-analysis. Soc Sci Med. 2017;192:125-33.

11. Murray JM, Brennan SF, French DP, Patterson CC, Kee F, Hunter RF. Mediators of behaviour change maintenance in physical activity interventions for young and middle aged adults: a systematic review. Ann Behav Med. 2018;52(6):513-29.

12. Hunter RF, Tully MA, Davis M, Stevenson M, Kee F. Physical activity loyalty cards for behavior change: a quasi-experimental study. Am J Prev Med. 2013:45(1):56-63.

13. Dallat MAT, Hunter RF, Tully MA, Cairns KJ, Kee F. A lesson in business: costeffectiveness analysis of a novel financial incentive intervention for increasing physical activity in the workplace. BMC Public Health. 2013;13(1):953.

14. Hunter RF, Brennan SF, Tang J, et al. Effectiveness and cost-effectiveness of a physical activity loyalty scheme for behaviour change maintenance: a cluster randomised controlled trial. BMC Public Health. 2016;16(1):618.

15. Department of Health. UK physical activity guidelines. 2011. [http://www.dh. Gov.Uk/en/Publicationsandstatistics/publications/ PublicationsPolicyAndGuidance/DH_128209]. Accessed Jan 2016.

16. Carson RT. Contingent valuation: a user's guide. Environ Sci Technol. 2000; 34:1413-8.

17. Miller N, Dollard J. Social learning and imitation. New Haven: Yale University Press; 1941.

18. National Institute for Health and Care Excellence (2012). Methods for the development of NICE public health guidance (third edition) [PMG4]. 6 Incorporating health economics. https://www.nice.org.uk/process/pmg4/ chapter/incorporating-health-economics. Accessed 29 November 2017.18.

19. Gough A, Prior P, Kee F, Hunter RF, on behalf of the PAL study team. Physical activity and behaviour change. The role of distributed motivation Crit Public Health. 2018; https://doi.org/10.1080/09581596.2018.1535169. 
20. Murray JM, Gough A, French DP, Tang J, Kee F, Hunter RF. Investigating whether engagement with the specific components of an internet-based intervention with financial incentives predicts physical activity outcomes and mediators for sedentary office-based employees: process analysis of the Physical Activity Loyalty (PAL) scheme, a cluster randomised controlled trial (under review).

21. Claxton K. The irrelevance of inference: a decision-making approach to the stochastic evaluation of health care technologies. J Health Econ. 1999;18(3): 341-64.

22. Deci EL, Ryan RM. Intrinsic Motivation and Self-determination in human behavior. New York: Plenum Press; 1985

23. Promberger M, Marteau TM. When do financial incentives reduce intrinsic motivation? Comparing behaviors studied in psychological and economic literatures. Health Psychol. 2013;32(9):950-7.

24. Bonell C, Jamal F, Melendez-Torres GJ, Cummins S. 'Dark logic': theorising the harmful consequences of public health interventions. J Epidemiol Community Health. 2015;69:95-8. https://doi.org/10.1136/jech-2014-204671.

25. Tannahill A, Kelly MP. Layers of complexity in interpreting evidence on effectiveness. Public Health. 2013;127(2):164-70.

26. Lilford RJ, Chilton PJ, Hemming K, Girling AJ, Taylor CA, Barach P. Evaluating policy and service interventions: framework to guide selection and interpretation of study end points. BMJ. 2010;341:c4413. https://doi.org/10. 1136/bmj.c4413.

27. Wilcox AJ. A positive approach to negative results. Epidemiology. 2014;25(2):165.

28. Conn VS, Hafdahl AR, Mehr DR. Interventions to increase physical activity among healthy adults: meta-analysis of outcomes. Am J Public Health. 2011; 101(4):751-8.

29. Puig-Ribera A, Bort-Roig J, González-Suárez AM, et al. Patterns of impact resulting from a "sit less, move more" web-based program in sedentary office employees. Zhang H, ed. PLoS One. 2015;10(4):e0122474.

30. Basu S, Kiernan M. A simulation modeling framework to optimize programs using financial incentives to motivate health behavior change. Med Decis Mak. 2016;36(1):48

Ready to submit your research? Choose BMC and benefit from:

- fast, convenient online submission

- thorough peer review by experienced researchers in your field

- rapid publication on acceptance

- support for research data, including large and complex data types

- gold Open Access which fosters wider collaboration and increased citations

- maximum visibility for your research: over $100 \mathrm{M}$ website views per year

At $\mathrm{BMC}$, research is always in progress.

Learn more biomedcentral.com/submissions 